\title{
INTERVENCIÓN PSICOSOCIAL EN ESPACIOS DE ALTA DIVERSIDAD: EXPERIENCIA INTERCULTURAL Y BUENA PRAXIS
}

\author{
Isaac Irán Cabrera ${ }^{1}$, Ángel Joel Méndez López ${ }^{2}$. \\ ${ }^{1}$ Universidad Central "Marta Abreu" de las Villas. Cuba \\ ${ }^{2}$ Universitat de València. España
}

\section{Resumen}

Los enfoques interventivos psicosociales en la reducción de sesgos intergrupales, pueden dividirse en dos ámbitos: intragrupales e intergrupales. En las primeras se encuentra el trabajo con ejemplares contraesterotípicos (Plant et al., 2009), las estrategias basadas en la disonancia cognitiva (Festinger, 1957, citado en Rodríguez, 2019) y la promoción de la empatía (Galinsky \& Moskowitz, 2000; Stephan \& Finlay, 1999). Como estrategias intergrupales se identifican las basadas en la hipótesis del contacto (Allport, 1954, citado en Rodríguez, 2019) y las basadas en la teoría de la categorización social o estrategias sociocognitivas (Tajfel \& Turner, 1979). La investigación se adscribió al modelo de investigación interventiva propuesto por Rothman y Thomas (1994) constituido por tres áreas: desarrollo de conocimiento, utilización de conocimiento y diseño y realización de intervenciones. El proceso se orientó específicamente en la tercera de estas áreas.

Se siguió un tipo de investigación cualitativa constructivointerpretativa, en cuyo marco se realizó una sistematización de experiencias. Se sigue la propuesta metodológica ofrecida por Jara (1994) desde la que se estructura la sistematización en cinco tiempos: el punto de partida (haber sido participante y tener registros de la experiencia), las preguntas iniciales (objetivo, objeto, eje de sistematización, fuentes, procedimientos), la recuperación del proceso vivido (reconstruir la historia, ordenar y clasificar la información), la reflexión de fondo (análisis, síntesis, interpretación crítica) y los puntos de llegada (formular conclusiones y comunicar los aprendizajes). Se consideraron los criterios de validación del material sistematizado propuestos por De Armas (2014): pertinencia social, factibilidad, aplicabilidad y rigor científico.

La intervención psicosocial en espacios de alta diversidad se estructuró mediante tres proyectos que emplean como estrategias de intervención psicosocial la coalición comunitaria, el grupo de aprendizaje y la sensibilización.

Palabras claves: calidad de vida, atención, memoria, adulto mayor

\begin{abstract}
Psychosocial interventional approaches in reducing intergroup biases can be divided into two areas: intragroup and intergroup. The former includes work with counterstereotype specimens (Plant et al., 2009), strategies based on cognitive dissonance (Festinger, 1957, cited in Rodríguez, 2019) and the promotion of empathy (Galinsky \& Moskowitz, 2000; Stephan \& Finlay, 1999). Intergroup strategies are those based on the contact hypothesis (Allport, 1954, cited in Rodríguez, 2019) and those based on the theory of social categorization or socio cognitive strategies (Tajfel \& Turner, 1979). The research was ascribed to the interventional research model proposed by Rothman and Thomas (1994), consisting of three areas: knowledge development, use of knowledge, and design and implementation of interventions. The process focused specifically on the third of these areas.
\end{abstract}

A type of constructive-interpretive qualitative research was followed, in which a systematization of experiences was carried out. The methodological proposal offered by Jara (1994) is followed from which the systematization is structured in five times: the starting point (having been a participant and having records of the experience), the initial questions (objective, object, systematization axis, sources, procedures), the recovery of the lived process (reconstructing history, ordering and classifying information), deep reflection (analysis, synthesis, critical interpretation) and points of arrival (formulating conclusions and communicating learning). The validation criteria of the systematized material proposed by De Armas (2014) were considered: social relevance, feasibility, applicability, and scientific rigor.

Psychosocial intervention in highly diverse spaces was structured through three projects that use the community coalition, the learning group and awareness as psychosocial intervention strategies.

Keywords: quality of life, attention, memory, older adult

\section{Correspondencia Ángel Joel Méndez López}

Departamento de Trabajo Social y Servicios Sociales de la Universitat de València, España

Email: menanjo@uv.es 


\section{RELACIONES INTERCULTURALES Y SUBJETIVIDAD}

Las relaciones interculturales, en el marco de la interacción grupal, se definen como formas específicas de relación social entre individuos, que interactúan en calidad de miembros de grupos culturalmente diversos, a partir de la reproducción y producción sociopsicológica de pertenencias y exclusiones grupales, mediadas por factores históricos, económicos y socioculturales (Cabrera-Ruiz, Rodríguez-González, \& Rodríguez-Fleites, 2016). La formación de pertenencias y exclusiones grupales, tiene lugar mediante procesos sociopsicológicos de apropiación de clasificaciones culturalmente disponibles, que proveen a los sujetos de auto-referencias, las cuales les permiten reconocerse como miembros de categorías sociales (nacionalidad, etnia, religión, género).

En las relaciones interculturales, queda acentuada la diversidad de origen como categoría de referencia, que deviene en diferenciación entre el endogrupo y el exogrupo. De esta manera, al bosquejar la interculturalidad desde las relaciones intergrupales, se pondera el proceso de identificación social, mediador de las dimensiones relacionales y simbólico-emocionales, en función de la membrecía grupal. El énfasis en la diferencia y la omisión de la igualdad, favorece los efectos excluyentes en detrimento de la integración. De esta forma, las relaciones interculturales, se constituyen como objeto de configuración de la subjetividad social.

Entendemos como subjetividad social precisamente, el sistema integral de configuraciones subjetivas (grupales o individuales) que se articulan en los distintos niveles de la vida social, implicándose de forma diferenciada en las distintas instituciones, grupos y formaciones de una sociedad concreta. Estas formas tan disímiles, guardan complejas relaciones entre sí y con el sistema de determinantes de cada sociedad concreta, aspectos que deben ser integrados y explicados por las Ciencias Sociales. En esta dirección encontramos que, las Ciencias Sociales no pueden contribuir a la reproducción de esquemas inoperantes de gestar subjetividad social en lo cotidiano de vida. Su hacer enriquecido se inserta en un enfoque integral del desarrollo donde no se subestime ninguna arista del fenómeno bajo análisis y donde no se encuadre o simplifique la realidad por la demanda de lo urgente o lo inmediato. Tampoco puede perder de vista a las personas, en tanto seres aportativos, actuantes, transformadores.

Por su parte, "la subjetividad se define como producción de sentidos y configuraciones subjetivos de la experiencia" (González-Rey, 2015, p.25). Es una producción simbólico-emocional, que emerge ante una experiencia vivida, a partir de la interrelación entre lo individual, contextual e histórico-social; que se configura recursivamente en los espacios sociales de la experiencia y en las personas (González-Rey 1997, 2006, 2007, 2011).

Para González- Rey (2009) la subjetividad está presente en todos los procesos y niveles de las producciones humanas, desde las individuales hasta las institucionales. Representa 
así, un sistema que tiene un momento organizativo en la personalidad como subjetividad individual, pero que a la vez existe en un sujeto en acción y relación, es el sujeto personal subjetivado de la acción social que participa en una multiplicidad de sujetos y espacios sociales.

La subjetividad social y la individual no son polos extremos, sino procesos tensionales que se configuran recíprocamente (Díaz-Gómez, González-Rey y Arias-Cardona, 2017). En los espacios sociales de la experiencia, todo lo social converge como una nueva configuración, que la coloca como nivel cualitativo diferenciado de los propios procesos que están en su génesis.

"Las configuraciones subjetivas individuales están configuradas en el espacio de la subjetividad social dominante donde esas individualidades transitan" (p.138).

A su vez, el sentido subjetivo, es "la unidad inseparable de los procesos simbólicos y las emociones en un mismo sistema, en el cual la presencia de uno de esos elementos evoca al otro, sin que sea absorbido por el otro" (González- Rey, 2003, p. 127). Toda experiencia vivida es sentida y se expresa subjetivamente por medio de su configuración subjetiva. El sentido es el momento procesual de la experiencia, por lo que siempre que emergen tienden a organizarse en el proceso mismo como una configuración subjetiva de la acción. La configuración subjetiva se define como forma de organización de los procesos de sentido. Existe como red de distintos desdoblamientos simbólicosemocionales que toman un sentido u otro según el contexto que está viviendo el sujeto (Díaz-Gómez, González-Rey y Arias-Cardona, 2017).

En el marco de un enfoque psicosocial de la interculturalidad y una concepción histórico-cultural de la subjetividad, se encuentra la producción científica de Cabrera (2011), Cabrera, Rodríguez, y Rodríguez (2016), Pérez-Borroto, Cabrera, y Rodríguez (2020). Estos referentes ubican las relaciones interculturales como objeto de configuración de la subjetividad social la cual, organizada como estereotipos y prejuicios transmitidos en procesos socializadores, reproduce la exclusión mediante el reconocimiento de la diferencia que promueve y justifica condiciones reales de discriminación hacia determinados grupos. De esta manera, se explicita el carácter mediador de la subjetividad. Rodríguez y Cabrera (2019) definieron la mediación por la subjetividad como las transiciones, a modo de movimiento dialéctico, de determinada contradicción social entre su configuración en los sistemas intersubjetivos asociados a la acción del sujeto y sus expresiones en los sistemas de relación que desde su actividad vive.

Las formas dinámicas de configuración subjetiva que mediatizan las relaciones intergrupales, definidas por estos autores, son estereotipos, prejuicios y discriminación. Los estereotipos constituyen una configuración subjetiva de creencias compartidas atribuidas a sí mismo u otras categorías o grupos sociales, a los cuales caracteriza de forma generalizada y diferenciadora en nosotros-los otros, a la vez que justifican el comportamiento intergrupal. 
Los prejuicios se definen como una actitud hacia los miembros de un grupo, cuyas tendencias evaluativas son fundamentalmente negativas. Definidas desde su existencia como subjetividad, las actitudes son predisposiciones y tendencias de comportamiento que tienen un componente único: sentidos subjetivos evaluativos de la realidad social (Cabrera, Rodríguez, \& Álvarez, 2020). Estos autores concuerdan en que el elemento más pernicioso del prejuicio es la discriminación; esta consiste en conductas de estigmatización y rechazo de otras personas por pertenecer a ciertos grupos sociales (Allport, 1962; Oskamp, 1991; Morales \& Páez, 1996).

Los resultados que se presentan en el presente trabajo, pertenecen a cuatro estudios enmarcados en la línea de investigación de las relaciones interculturales desde un enfoque psicosocial y una concepción histórico-cultural de la subjetividad desarrollada en la Universidad Central "Marta Abreu" de las Villas (UCLV), un espacio marcado por una alta diversidad.

Esta institución educativa cubana del nivel de educación superior cuenta, desde 1961, con una experiencia de inclusión de estudiantes extranjeros en el pregrado y, desde 1970, con un proyecto de internacionalización que ha permitido el desarrollo institucional y la promoción de la Educación Superior en Cuba hacia diversas latitudes. Hasta la fecha, esta universidad ha graduado 2239 estudiantes de diferentes nacionalidades. Los países con mayor cantidad de estudiantes graduados son Angola, Etiopía, Vietnam y Ghana, aunque el universo con el que se ha venido trabajando, va más allá de la patencia de sujetos representando estas nacionalidades.

\section{FUNDAMENTOS TEÓRICO-METODOLÓGICOS EN ESTUDIOS DE SUBJETIVIDADES EN LAS RELACIONES INTERCULTURALES}

La subjetividad se manifiesta en un proceso continuo que "no se devela de forma inmediata ante el observador; sus procesos y formas de organización tienen que ser construidos a partir de innumerables formas de expresión" (Rodríguez-González, 2015, p.14). La epistemología cualitativa para el estudio de la subjetividad, se define por tres características esenciales, según refiere González-Rey (1997, 2000, 2006, 2013): el conocimiento tiene un carácter constructivo interpretativo, la investigación es un proceso de comunicación y lo singular es relevante como nivel legítimo de la producción del conocimiento.

El saber nunca es un reflejo simplista o meramente lineal de lo externo, porque el dato no existe fuera de los significados, sino que es ya un concepto humano que simplifica el mundo. El investigador es un sujeto creador que emplea su imaginación en el desarrollo de la ciencia. La ciencia es proveedora de sistemas de significación, para organizar nuevas prácticas y funcionan porque generan la capacidad de significar nuevas experiencias.

El conocimiento, en los seres humanos, es un proceso dialógico, de comunicación en el que se propicia la calidad de la expresión de un ser humano. Esto es esencial para la emergencia del sujeto y su subjetividad. Por otra parte, el caso singular es una fuente legítima del conocimiento, a partir de su pertinencia y aporte al sistema teórico producido 
en la investigación, en el proceso permanente de implicación intelectual del investigador. La singularidad tiene valor por el carácter diverso del ser humano y de los espacios de vida social, según su organización en la cultura.

Las ciencias del ser humano tienen que reflejar la realidad desde lo histórico. Es muy heterogénea la sociedad y por ello es indispensable valorar el sentido subjetivo para el individuo. El sentido subjetivo es clave para comprender la subjetividad, siendo ésta, parte componente (constituyente) de cualquier estructura individuo-grupo-sociedad. La ciencia no puede alejarse de lo que sienten y vivencian las personas en la cotidianeidad de su existencia, menos aún en un contexto social complejo, interconectado y multimediatizado. Su integración con lo popular, debe generar niveles de transparencia que produzcan consensos y crecimientos compartidos. Los estudios científicos deben encaminarse hacia la búsqueda de resultados integrales, donde el ser humano no desaparezca bajo ningún concepto. La ciencia al servicio del verdadero desarrollo societal, está en la obligación de rescatar y redimensionar a la persona desde sí misma.

Muchos análisis científicos hoy se mueven sobre la cuerda de lo enajenante y esa enajenación que aísla a la ciencia de la realidad cotidiana y, por consiguiente, de las personas que construyen y producen dicha cotidianeidad, idealiza la ciencia y la hace inoperante, disfuncional, estéril e inútil para entender adecuadamente cada fenómeno que discurre en la realidad. Las racionalidades externas no pueden continuar manipulando los procesos sociales; urge refuncionalizar la cosificación del mundo. La ciencia debe fomentar felicidad cotidiana y no generar ansiedades, al igual que no puede legitimar intereses de poder.

La investigación constructiva-interpretativa es el tipo de investigación derivado de esta articulación epistemológica. Tiene como características principales: constituir un proceso relacional-dialógico; la conjunción de la aplicación de los instrumentos y la producción de la información como momentos inseparables; el desdoblamiento entre instrumentos en el proceso de construcción de la información; el carácter rector de las ideas en el desarrollo del modelo teórico; la cualidad de sujetos del proceso de investigación tanto del investigador como de los participantes con funciones e intereses diferentes.

Este tipo de diseño, enfatiza en el desarrollo de modelos teóricos como objetivo principal de la investigación (González-Rey y Mitjáns, 2016). Según estos autores, un modelo teórico constituye una construcción teórica que tiene en lo empírico un momento esencial para su construcción, con capacidad de desarrollo progresivo de hipótesis y nuevos indicadores sobre la información empírica.

"El curso de una investigación cualitativa constructivo-interpretativa, es un proceso hipotético que puede ser negado por la emergencia de nuevos indicadores que refuten el camino dominante de la construcción teórica hasta ese momento" (González-Rey y Mitjáns, 2016, p.10). Constituye un proceso de construcción teórica progresivamente compleja que integra, en significados nuevos, una diversidad de expresiones de los sujetos participantes, que aisladamente no tendrían valor para el conocimiento. 
Díaz-Gómez, González-Rey y Arias-Cardona (2017) exponen que en este tipo de investigación no se niega la cantidad, solo que ella es un significado, no es una conclusión; no es necesario el criterio de muestreo poblacional, sino la representación diversa de la población con la que se quiere dialogar; y, la generalización se presenta en términos del modelo teórico. El investigador, en una constante reflexión, genera significados sobre los datos y generaliza, no de forma inductiva, sino desde su referente teórico. Ello permite que el modelo, como herramienta de acceso a lo empírico, gane nuevas capacidades de inteligibilidad en la medida en que se extiende y multiplica en una línea de investigación.

En el marco de la línea de investigación de las relaciones interculturales desde un enfoque psicosocial y una concepción histórico-cultural de la subjetividad, se desarrolló la comprensión de la mediación por la subjetividad como las transiciones, a modo de movimiento dialéctico, de determinada contradicción social entre su configuración en los sistemas intersubjetivos asociados a la acción del sujeto y sus expresiones en los sistemas de relación que desde su actividad vive. La mediación por la subjetividad constituye la participación de la subjetividad en la reproducción/producción de las contradicciones configuradas como subjetividad social (institucional, organizacional, comunitaria, grupal) y su objetivación en el comportamiento social.

Las mediaciones se encuentran en el trasfondo de una expresión singular/particular, que se nos presenta como inmediata, pero que es resultante de una ligazón de factores sociohistóricos, económicos y biográficos que se actualiza en la acción y como configuración subjetiva y que produce/reproduce el sistema de contradicciones en que está inmersa. Como expresa Pérez Yera (2009) los sistemas de relación y las subjetividades no pueden ser comprendidas "si no se conocen, se descubren, se hacen explícitas, las mediaciones que se encuentran por detrás, como ocultas en lo inmediatamente dado" (p. 256). Tal posicionamiento es ilustrado en el esquema 1.

\section{Figura 1}

Representación gráfica de la comprensión de las mediaciones por la subjetividad.

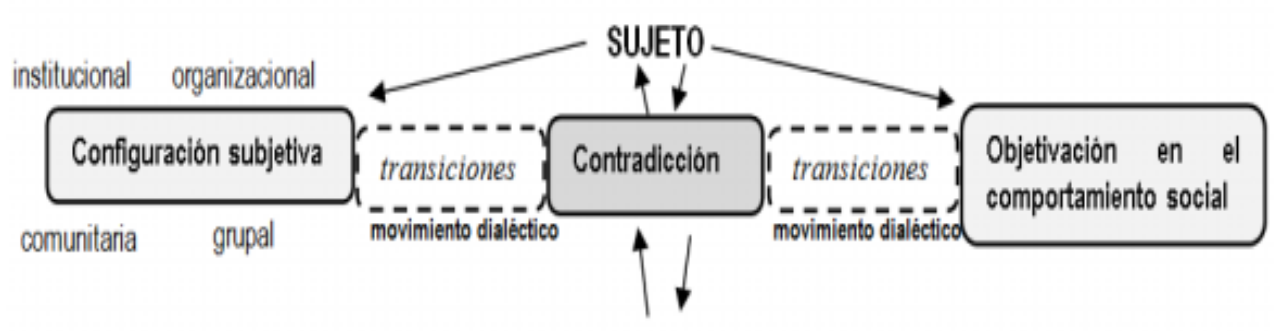

sistemas de acción y relación

\section{Fuente: Elaboración propia}

Los estudios realizados, se sustentaron en estos fundamentos teórico-metodológicos. Dos de los cuatro estudios, estuvieron dirigidos a develar estereotipos en las relaciones 
interculturales desde estudiantes cubanos y extranjeros. Ambos se realizaron en 2015. El tercero, realizado en 2017, se orientó al estudio de la integración intercultural como proceso subjetivado en grupos escolares. El cuarto estudio, ejecutado en 2018, se dirigió a develar la permanencia en el tiempo de estereotipos en las relaciones interculturales.

La realización longitudinal de los estudios, permitió arribar al punto de saturación y generar nuevas capacidades de inteligibilidad en el modelo teórico debido a la consideración, en sus diseños teórico-metodológicos, de nuevas contradicciones en el estudio de las relaciones interculturales. En el 2015, se identificó la ausencia de estudios orientados a develar estereotipos interculturales en los estudiantes universitarios en Centros de Educación Superior cubana.

El modelo teórico comprensivo de los estereotipos los definió como forma de subjetividad social que se expresa como configuración de creencias (producciones de sentido) respecto a ciertos atributos referidos a uno mismo y al otro, por la pertenencia a categorías sociales que posibilitan el reconocimiento y la diferenciación como grupo. Desde este modelo se evidenció la presencia de tres núcleos de sentido en la configuración de estereotipos en las relaciones interculturales: percepción de los grupos etnoculturales, diferencias e inequidades emergentes del origen etnocultural en la formación y proyección profesional y discriminación por la pertenencia etnocultural.

En 2017 se declaró la ausencia de estudios relacionados con la integración intercultural desde un Enfoque Histórico-Cultural, que permitan comprender la mediación de las configuraciones subjetivas en las dinámicas de integración en el contexto universitario. El modelo teórico, se fundamentó en la construcción de tres núcleos de sentido asociados al reconocimiento de cualidades personales en los procesos de integración intercultural, el grupo escolar en los procesos de integración intercultural y la dinámica de las relaciones interculturales. Estos núcleos de sentido pretendían comprender la subjetivación de los procesos de integración intercultural en los grupos escolares estudiados.

El primer núcleo de sentido estuvo constituido por dos indicadores distintos en función de la atribución de cualidades personales, por parte de estudiantes extranjeros o por parte de estudiantes cubanos. El segundo estuvo constituido por los indicadores desempeño académico y relaciones en el grupo escolar en función de la actividad de estudio. El tercero, por los indicadores contradicciones reconocidas por los estudiantes en los procesos de integración intercultural, estrategias multiculturalistas y valoración de las relaciones interculturales.

En 2018 se asumió la carencia de estudios centrados en la permanencia en el tiempo de estereotipos en las relaciones interculturales en la Universidad Central de Las Villas, en el período 2015-2018. Los estereotipos se configuraron en torno a rasgos que diferencian a los grupos etnoculturales en cuanto a aspectos físicos, características personológicas, conducta social y aficiones. La desigualdad por la pertenencia al grupo social se expresó mediante los indicadores posición económica y competencias en el proceso de formación profesional; los cuales colocan en posición de ventaja o desventaja a cubanos y extranjeros. 


\section{ASPECTOS METODOLÓGICOS PARA EL ESTUDIO DE LA SUBJETIVIDAD EN LAS RELACIONES INTERCULTURALES}

La construcción e interpretación de la realidad social estudiada, desde la construcción de modelos capaces de generar inteligibilidad respecto a mediaciones por la subjetividad en las relaciones interculturales, se caracterizó por la flexibilidad, carácter abierto en la toma de decisiones de forma continua y recurrente conforme a la aproximación y las necesidades del estudio. Los estudios implicaron momentos de conceptualización, categorización y reflexión, en los que se modificó y redefinió la información en aras de un mejor acercamiento científico. El carácter emergente del diseño de investigación se expresó particularmente en cuanto al problema y objetivos de investigación, la relación con la teoría y el momento empírico y la selección de la muestra. En la tabla 1 se expone el objetivo general de cada estudio.

\section{Tabla 1}

Estudios de subjetividades en las relaciones interculturales en la universidad central "Marta Abreu” de las villas.

\begin{tabular}{|c|c|c|}
\hline Título & Año & Objetivo General \\
\hline $\begin{array}{l}\text { Estereotipos en las relaciones } \\
\text { interculturales: una mirada desde } \\
\text { estudiantes cubanos de la UCLV. }\end{array}$ & 2015 & $\begin{array}{l}\text { Develar estereotipos configurados en } \\
\text { estudiantes cubanos de la UCLV en sus } \\
\text { relaciones interculturales. }\end{array}$ \\
\hline $\begin{array}{l}\text { Estereotipos en las relaciones } \\
\text { interculturales: una mirada desde } \\
\text { estudiantes universitarios } \\
\text { extranjeros de la UCLV }\end{array}$ & 2015 & $\begin{array}{l}\text { Conocer estereotipos configurados en } \\
\text { estudiantes extranjeros de la UCLV en sus } \\
\text { relaciones interculturales. }\end{array}$ \\
\hline $\begin{array}{l}\text { Integración intercultural como } \\
\text { proceso subjetivado: estudio en } \\
\text { grupos escolares de la UCLV }\end{array}$ & 2017 & $\begin{array}{l}\text { Producir la configuración subjetiva de la } \\
\text { integración intercultural en grupos } \\
\text { escolares de la UCLV. }\end{array}$ \\
\hline $\begin{array}{l}\text { Entre la integración y la } \\
\text { exclusión: permanencia en el } \\
\text { tiempo de estereotipos en las } \\
\text { relaciones interculturales en la } \\
\text { UCLV }\end{array}$ & 2018 & $\begin{array}{l}\text { Constatar la permanencia en el tiempo de } \\
\text { estereotipos que median los procesos de } \\
\text { integración-exclusión en las relaciones } \\
\text { interculturales en la UCLV en el período } \\
2015-2018 \text {. }\end{array}$ \\
\hline
\end{tabular}

Fuente: Elaboración propia

\subsection{Selección de los participantes}

La selección de los participantes se apoyó en el muestro intencional opinático (RuizOlabuénaga, 1999) y el muestreo por juicio (Mejía, 2000). Ambos tipos de muestreos, consideran la selección de los participantes con un carácter dirigido o no probabilístico, en función de las intenciones del investigador. De esta manera, se garantizaron la cantidad 
o saturación y la calidad o riqueza de la información. Su modalidad de muestreo opinático, permitió seleccionar a los participantes, según un criterio estratégico personal.

El principio de pertinencia y adecuación (Sandoval, 1996) se cumplió mediante la identificación de aquellos escenarios y participantes que pudieran aportar datos suficientes para desarrollar una completa y rica descripción del fenómeno. Se partió de un muestreo de escenario dentro del contexto de investigación, la Universidad Central de Las Villas, como espacio de confluencia intercultural.

El muestreo de escenario permitió identificar extranjero y cubano como categorías sociales supraordenadas configuradoras de grupos de referencia con respecto a las relaciones interculturales. De este modo se articularon dos ejes muestrales, estudiantes extranjeros y estudiantes cubanos, a los que se les aplicó, respectivamente, una estrategia de máxima variación o de heterogeneidad entre grupos para mostrar distintas perspectivas y representar la complejidad del fenómeno estudiado (Valles, 1999).

Se consideraron como criterios: ser estudiantes cubanos o ser estudiantes universitarios extranjeros, pertenecientes a uno de los grupos etnoculturales declarados en la investigación; pertenecer a una de las carreras por ramas de la ciencia consideradas de interés; provenir de un contexto áulico con presencia de estudiantes extranjeros o de un contexto áulico con ausencia de estudiantes extranjeros; contar con un tiempo superior a un curso académico interactuando en el contexto; ser accesibles en el momento de la investigación; aceptar participar de forma voluntaria en la investigación; así como brindar el consentimiento informado oral para participar en la investigación.

Las dos experiencias dirigidas a develar estereotipos en las relaciones interculturales desde estudiantes cubanos y extranjeros contaron con un total de 105 participantes, 63 cubanos y 42 extranjeros (20 africanos, 9 latinos, 7 caribeños y 6 asiáticos). Los estudiantes cubanos fueron seleccionados por ramas de la ciencia con mayor número de estudiantes extranjeros y diversidad de grupos etnoculturales en su matrícula. Provenían de las carreras Licenciatura en Psicología, Ingeniería Eléctrica, Licenciatura en Ciencias de la Computación, Medicina Veterinaria y Zootecnia y Licenciatura en Economía. Los estudiantes extranjeros provenían de Angola, el Caribe, Latinoamérica y Asia. Predominó la presencia del sexo femenino (60) frente al masculino (43).

La experiencia Integración intercultural como proceso subjetivado: estudio en grupos escolares de la Universidad Central "Marta Abreu” de Las Villas asumió como muestra los grupos segundo año de Licenciatura en Derecho que cuenta con 72 estudiantes de Cuba, Angola y Guinea Ecuatorial y el tercer año de Ingeniería en Telecomunicaciones y Electrónica que cuenta con 50 estudiantes de Cuba, Angola y Congo. Como estrategia para la selección de los participantes se siguieron los criterios: grupos escolares con mayor diversidad de relaciones interculturales; grupos escolares constituidos con un tiempo superior a un curso académico de interrelación en el contexto; ser accesibles durante la investigación. No se declaró la composición por género.

En la experiencia Entre la integración y la exclusión: permanencia en el tiempo de estereotipos en las relaciones interculturales en la Universidad Central de Las Villas la 
muestra quedó conformada por 140 estudiantes cubanos y extranjeros pertenecientes a las facultades Matemática, Física y Computación, Ciencias sociales, Ingeniería Eléctrica, Ciencias Económicas, Construcciones y Química Farmacia (20 estudiantes por facultad excepto Ciencias Sociales - 40 estudiantes). La distribución de los participantes por año académico fue variada, la mayor concentración de estudiantes se encuentra en el tercer y cuarto año de la carrera.

En función de la nacionalidad, se contó con la participación de 97 cubanos que representan un $69,3 \%, 1$ europeo que representó un $0,7 \%, 34$ africanos para una representación de un 24,3\%, 2 asiáticos que representan un 1,4\%, 1 latino y caribeño para un $0,7 \%$ y 5 árabes que representan un 3,6\%. Contó con la participación de 64 representantes del sexo femenino para un $45,7 \%$ de la muestra y una representación de 76 participantes del sexo masculino para una representación de un 54, $3 \%$ de la muestra. Del total de representantes del sexo femenino 49 son cubanas, 12 son africanas, 1 asiática y 2 árabes. Del total de representantes del sexo masculino 48 son cubanos, 1 europeo, 22 africanos, 1 asiático 1 latino y caribeño y 3 árabes.

\subsection{Métodos, técnicas y procedimientos}

La selección de los instrumentos respondió a la unidad cosmovisiva entre el enfoque metodológico y el diseño de la investigación, por lo que se orientan la expresión abierta y comprometida de los sujetos, en tanto constituyen herramientas interactivas que le facilita al investigador el uso de estímulos y situaciones según los objetivos planteados. No siguieron reglas estandarizadas para su construcción por la complejidad del acceso a la producción de sentidos subjetivos en el que tiene lugar el proceso de naturalización de los espacios y fenómenos de la subjetividad social.

Los instrumentos seleccionados, representaron "una fuente de información separada de cualquier sistema de categorías preestablecidas orientadas a significar esa información" (González-Rey, 2006, p.67); sin embargo, forman un sistema único en el que los significados producidos de forma parcial por un instrumento concreto constituyen hipótesis dentro del sistema completo de la información producida.

Se empleó el análisis de documentos para conocer los antecedentes del contexto en relación a la presencia estudiantes extranjeros y el funcionamiento institucional. Se analizaron documentos de naturaleza institucional y formal como: informes estadísticos de estudiantes extranjeros por facultad, carrera, año de estudio y tipo de curso; matrícula por países; informe de nuevos ingresos y el proyecto de acompañamiento al estudiante extranjero.

La entrevista semi-estructurada constituyó una guía flexible que incluyó una serie de temas preestablecidos que fueron manejados de acuerdo con la información emergente. Se realizaron con el objetivo de conocer el estado actual de las relaciones interculturales en el contexto de investigación al jefe de departamento de atención a extranjeros y profesores que atienden a estudiantes extranjeros por facultad.

El completamiento de frases constituyó una adaptación de la versión para el diagnóstico educativo intercultural de Cabrera y Gallardo (2011). Su elaboración por el 
equipo de investigación estuvo determinada por la construcción de frases que no definen su valor por el contenido explícito que las define, por lo que los sujetos pueden expresar sentidos subjetivos diversos ante cada una de ellas.

La técnica de lista de adjetivos empleado durante la investigación se conformó en el equipo de investigación a partir de un listado de 105 adjetivos para identificar a los grupos etnoculturales latinos, caribeños, africanos, cubanos, árabes y europeos. Posteriormente la técnica fue sometida a un pilotaje. Como resultado del proceso la lista quedó estructurada, finalmente por solo 72 adjetivos.

Desde el formato grupal se emplearon la sociometría, el grupo de discusión, el grupo de enfoque, y observación científica de procesos grupales. El test sociométrico se basó en cuatro preguntas cortas y ordenadas por cada contenido. Se mantuvo un lenguaje claro y sencillo en relación a las circunstancias y experiencias grupales.

Para la realización de las actividades con formato grupal se contó con un equipo de coordinación compuesto por coordinador/investigador e investigadores auxiliares y una guía de planificación de sesión. Se propició la emergencia de una comunicación caracterizada por la apertura y no directividad. Se negoció con los sujetos la posibilidad de registrar la información mediante grabaciones de audio para asegurar su validez.

La observación científica de procesos grupales comprendió la captación de un conjunto de procesos mediadores presentes en la dinámica de los grupos. Estos procesos, seleccionados como unidades de observación, fueron la pertenencia, la pertinencia, la comunicación, la cooperación y el clima de interacciones; sustentados en la propuesta de Rivero (2012). Para su realización se precisó de la incorporación de los investigadores/observadores a las referidas dinámicas y se empleó como técnica la observación abierta y estructurada (Hernández, Fernández \& Baptista, 2014). La tarea de observación implicó tanto acciones de registro de datos como de interpretación a partir de los referentes epistémicos, teóricos y metodológicos del equipo de investigación.

La construcción de la información en el marco del planteamiento epistemológico y el posicionamiento en un enfoque histórico cultural de la subjetividad constituyó un reto; debido, en primer lugar, a la carencia de materiales didácticos referentes al proceso constructivo-interpretativo del conocimiento en el estudio de la subjetividad, y especialmente, de las formas de subjetividad social, carencia que es reconocida por González-Rey (2006), autor de la concepción configuracional.

En segundo lugar, fue un reto el correcto posicionamiento en cuanto a la relación sentido y configuración psicológica. Para ello fue necesaria la ruptura con la linealidad categorías subcategorías y la comprensión del sentido como forma de subjetivación de la experiencia social; lo que nos condujo a la identificación del núcleo de sentido como organización de sentidos configuradores de formas de subjetividad social, en este caso los estereotipos.

El proceso de construcción de la información se realizó mediante el análisis de contenido, técnica que permitió formular inferencias reproducibles y válidas a partir de los datos empíricos, el contexto de los datos y la forma en que el conocimiento de los 
investigadores los condujo al análisis e interpretación la realidad (Krippendorff, 1980). Las fases y procedimientos seguidos para el análisis de contenido están sustentadas en la sistematización realizada por Cabrera (2009).

La lógica configuracional, como organización del proceso constructivo-interpretativo, implicó la construcción progresiva por parte del equipo de investigadores de las categorías y su relación en el modelo comprensivo del problema estudiado. El uso del programa computacional para análisis cualitativo de datos Atlas, permitió la elaboración de gráficos para la visualización de los resultados. Se empleó la herramienta Tabla de coocurrencia, para visualizar los códigos co-ocurrentes con un código seleccionado como pauta. La herramienta Tabla de códigos por documentos primarios permitió la cuantificación de las veces en que se empleó determinado código.

Las investigaciones aseguraron su calidad mediante el cumplimiento de requisitos éticos y técnicos que sustentaron la transparencia y el rigor observados desde la formulación hasta la culminación del proceso investigativo. Los criterios de calidad, confianza y autenticidad de los hallazgos que se consideraron son sistematizados por Sandoval (1996).

Para cumplir con el criterio objetividad-confirmabilidad fueron descritos los métodos y procedimientos empleados en el estudio; se siguió una secuencia que es posible reconstruir por los lectores para obtener, procesar, condensar, transformar y mostrar los datos; se estableció un vínculo explícito entre las conclusiones planteadas y los datos condensados; se presentó un registro detallado y suficiente de los métodos y procedimientos utilizados en la investigación, así como se consideraron las hipótesis y conclusiones rivales.

Cumplir con el criterio confiabilidad-auditabilidad implicó la elaboración de preguntas de investigación claras, el planteamiento del diseño de investigación congruente con ellas, la descripción del papel y posición del equipo de investigadores dentro de la investigación, la valoración del paralelismo significativo de los hallazgos a través de las fuentes de datos y la especificación de los paradigmas básicos y constructos analíticos.

Además, se recolectaron los datos a través de un apropiado muestreo de escenarios, tiempos e informantes, se desarrollaron protocolos comparables entre sí por parte de los diferentes investigadores involucrados en el proyecto de investigación y se realizó una revisión de la codificación elaborada y un control de calidad de los datos.

Para dar cumplimiento al criterio validez interna-credibilidad-autenticidad se valoró la significatividad y riqueza de contexto de las descripciones presentadas; se realizó una reconstrucción plausible y convincente; se valoró la producción de conclusiones convergentes a partir de la triangulación entre métodos complementarios y fuentes de datos; se explicó coherentemente la obtención de datos divergentes a partir de la triangulación, se mostró adecuadamente la relación entre los datos presentados y las categorías de análisis, se explicitó la lógica y reglas seguidas para confirmar inferencias emergentes y se sometió a consideración de los informantes las conclusiones presentadas. 
Para cumplir con el criterio validez externa-transferibilidad-pertinencia fueron descritas las características de la selección o muestreo original de personas, escenarios y procesos; se establecieron claramente los límites de generalización mediante la definición explícita de los alcances y las limitaciones de la investigación; se incluyó una descripción completa y suficiente de los hallazgos para que los lectores puedan evaluar el potencial de transferibilidad y pertinencia a otros escenarios y se estableció un vínculo congruente entre los hallazgos y la teoría en la que se fundamentan.

Cumplir con el criterio utilización-aplicación-orientación a la acción implicó el planteamiento de los hallazgos de manera accesible intelectual y físicamente para los potenciales usuarios y de forma que se les estimule a proponer hipótesis orientadoras para la acción futura. Además, fueron presentados hallazgos que tienen un efecto catalizador dirigido a acciones específicas.

\section{CONSIDERACIONES FINALES}

La investigación constructivo-interpretativa, hizo viable la reconstrucción crítica del modelo teórico y ampliar sus capacidades de inteligibilidad. Los elementos constitutivos del modelo reconstruido fueron: configuraciones subjetivas, transiciones en que se expresa la contradicción en un acto dialógico-reflexivo generado por la investigación y sus expresiones en los sistemas de acción y relación.

Se constató el funcionamiento del origen nacional o regional como categoría de referencia en torno a la que se configuran identidades sociales y que deviene en diferenciación entre endogrupo y exogrupo (Huici, 2012; Turner, 1990; Tajfel, 1984). El proceso de categorización se expresó con dinamismo durante las sesiones, se movía desde nacionalidades, estudiantes financiados o autofinanciados, mujeres, las categorías regionales anteriormente expuestas, hasta la simple distinción cubano-extranjero. No obstante, el discurso se mostró prolífero y con alto consenso, por lo que se constató el funcionamiento de las categorías regionales en la diferenciación intergrupal. Se apreció la tendencia a otorgar a cada categoría social una esencia subyacente (Estrada et al., 2004).

En la construcción de las configuraciones subjetivas de la diferencia y la identidad se constató la funcionalidad de las dimensiones sociabilidad, moralidad y competencias (Fiske et al., 2002; Leach et al., 2007; Cuadrado et al., 2017). Los índices sociabilidadafectividad, moralidad y capacidad instrumental son predominantes en la subjetivación de la diferencia, debido a que articularon mayor cantidad de sentidos.

La alternancia entre la superioridad e inferioridad de estatus para una misma categoría nacional o regional en el contexto universitario cubano influyó en la ausencia de un marcado favoritismo endogrupal. Un ejemplo de ello se encuentra en el grupo cubano que, si bien es el mayoritario y autóctono en el contexto, adquiere un estatus de inferioridad en función de la carencia de recursos económicos y tecnológicos.

El posicionamiento en la subjetividad y en la epistemología para su estudio (DíazGómez et al., 2017) amplió las miradas hacia el sujeto capaz de producir alternativas de 
subjetivación que se expresan como críticas, cuestionamientos, resistencia y autocríticas ante experiencias vividas que se reconstruyen en procesos dialógicos y reflexivos. Esta aproximación se considera relevante para el planteamiento de procesos de transformación social pues no se limita a aprehender al sujeto reproductor de estereotipos y prejuicios.

Los estudios permitieron la recuperación de los desdoblamientos individuales y su articulación en la construcción de configuraciones subjetivas alternativas. Esto se debió a su consideración como necesarios en los procesos de intervención en tanto "forman y extienden nuevos núcleos de producción de la subjetividad social a nivel institucional" (González-Rey, 2015, p.24).

Con la reconstrucción de los datos se constató en sujetos de ambos géneros la expresión de estereotipos, así como emergencias de alternativas de subjetivación ante ellos. Además, se develó que la diferenciación por género, con referencia a las mujeres al interior de las categorías nacionales o regionales, aparece cuando se expresan adjetivos asociados a la apariencia física, el cuidado de la estética, la belleza o la falta de estas características. Al referirse a las mujeres cubanas el alumnado angolano las estereotiparon mediante la falta de decencia, especialmente al vestir. Aunque de forma explícita emergen críticas al machismo, estos contenidos latentes fundamentan la necesidad de incluir el enfoque de género en los procesos de intervención.

Los resultados aportaron nuevos argumentos a la afirmación de que los contactos interculturales deben ser analizados con énfasis en las relaciones de poder establecidas en cada sociedad que sirven de marco a la producción de la diferencia y la identificación. La asimetría de poder se constató vinculada al peso demográfico y a factores económicos.

Arroyo (2013) plantea que la diversidad constituye una problemática cuando expresa desigualdades que surgen porque, por un lado, determinados sujetos o grupos alcanzan en desigual medida objetivos sociales y culturales; lo que, por otro lado, los enfrentan a aquellos con menores posibilidades de acceso. Escarbajal (2010) afirma que fácilmente se es consciente de que hay diferencias económicas, sociales y políticas que verdaderamente separan a las personas; no el color de la piel, la religión o el idioma.

Los resultados presentados orientan la transformación social hacia la promoción de oportunidades para el acceso equitativo de los grupos e individuos a los bienes y servicios que brinda la sociedad. Su correlato para los sujetos se observará en la oportunidad de insertarse en el proceso de toma de decisiones de manera activa y directa; no solo desde la posición de beneficiarios sino como actores del cambio social con capacidad para proyectar alternativas de vida.

\section{REFERENCIAS BIBLIOGRÁFICAS}

Arroyo González, M. (2013). La Educación Intercultural: un camino hacia la inclusión educativa. Revista de Educación Inclusiva, 6 (2), 144-159. https://dialnet.unirioja.es/servlet/articulo? codigo $=4335836$ 
Cabrera, I. (2009). El análisis de contenido en la investigación educativa: propuesta de fases y procedimientos para la evaluación de la información. Revista de Pedagogía Universitaria, 14(3), 71-93.

Cabrera, I. (2011). Educación intercultural del estudiante universitario: modelo desde la labor educativa del año académico. (Tesis en opción al Grado científico de Doctor en Ciencias Pedagógicas), Universidad Central "Marta Abreu" de Las Villas, Centros de Estudios de Educación.

Cabrera, I., Rodríguez González, D. R., \& Álvarez, R. (2020). Subjetividad, mediaciones y sociedad. En D. Pañella Álvarez \& I. I. Cabrera (coords.). Dinámicas subjetivas en la Cuba de Hoy, 7-19. La Habana: ALFEPSI

Cabrera, I., Rodríguez, D. R., y Rodríguez, L. (2016). Estereotipos en las relaciones interculturales. Hacia la construcción de un modelo comprensivo desde una concepción histórico cultural de la subjetividad. Santa Clara: Feijóo.

Cabrera, I., y Gallardo, T. (2011). Educación intercultural: Aproximaciones teóricas para una propuesta en la universidad cubana. Santa Clara: Feijóo.

Cuadrado, I., López-Rodríguez, L., \& Navas, M. (2017). "Si eres moral y competente adoptaré elementos de tu cultura": el rol mediador de las emociones positivas en el proceso de aculturación de inmigrantes ecuatorianos en España. Universitas Psychologica, 16 (5), 1-14. https://doi.org/10.11144/Javeriana.upsy16-5.mcae

Díaz-Gómez, A., González-Rey, F. \& Arias-Cardona, A.M. (2017). Pensar el método en los procesos de investigación en subjetividad. Rev. CES Psicol., 10 (1), 129-145

Escarbajal Frutos, A. (2010). Educación inclusiva e intercultural. International Journal of Developmental and Educational Psychology, 3 (1), 411-418. http://www.redalyc.org/articulo.oa?id=349832326044

González-Rey, F. (2015) Los estudios psicosociales hoy: aportes a la intervención psicosocial. En J. E. Moncayo y A. Díaz (eds.). Psicología Social Crítica e Intervención Psicosocial. Reflexiones y experiencias de investigación. 21-35. Cali: Universidad de San Buenaventura

González-Rey, F. \& Mitjáns, A. (2016) Una epistemología para el estudio de la subjetividad: sus implicaciones metodológicas. Psicoperspectivas. Individuo y Sociedad, 15 (1), 5-16

González-Rey, F. (1997). Epistemología cualitativa y subjetividad. La Habana: Editorial Pueblo y Educación

González-Rey, F. (2000). Investigación cualitativa en psicología. Rumbos y desafios. México, D.F: International Thomson Editores

González-Rey, F. (2006). Investigación cualitativa y subjetividad. Guatemala: Oficina de Derechos Humanos del Arzobispado de Guatemala. 
González-Rey, F. (2013). La subjetividad en una perspectiva cultural-histórica: avanzando sobre un legado inconcluso. Revista de Ciencias Sociales, 11, 19-42.

Mejía, J. (2000). El muestreo en la investigación cualitativa. Revista de Investigaciones Sociales, 5.

Morales, J. F. \& Páez, D. (1996). Estereotipos, discriminación y relaciones intergrupo en España y Latinoamérica. En R. Bourhis y J. P. Leyens (Eds.), Estereotipos, discriminación y relaciones entre grupos. Madrid: McGraw Hill.

Oskamp, S. (1991). Attitudes and opinions. Englewood Clifs, Nueva Jersey: Prentice Hall.

Pérez Yera, A. (2009) La mediación por la subjetividad del autodesarrollo comunitario. En C. de autores. El desarrollo local comunitario. Desafios actuales para América Latina. (217-256). Santa Clara: Feijóo

Pérez-Borroto, S., Cabrera, I. I., \& Rodríguez González, D. R., \& (2020). Permanencia en el tiempo de estereotipos interculturales. (122-135). En D. Pañella Álvarez \& I. I. Cabrera (coords.). Dinámicas subjetivas en la Cuba de Hoy. La Habana: ALFEPSI

Rodríguez González, D. R. \& Cabrera, I. I. (2019). Mediaciones por la subjetividad del autodesarrollo comunitario en las relaciones interculturales. Estudios en la Universidad Central "Marta Abreu” de Las Villas. Santa Clara: Feijóo

Rodríguez-González, D. (2015). Estereotipos en las relaciones interculturales: una mirada desde estudiantes cubanos de la Universidad Central «Marta Abreu» de Las Villas. Trabajo de Diploma. Facultad de Psicología. Universidad Central "Marta Abreu" de Las Villas. Santa Clara

Ruiz-Olabuénaga, J. (1999). Metodología de la investigación cualitativa (2da. ed.). Bilbao: Universidad de Deusto

Sandoval, C. (1996). Investigación cualitativa. Bogotá: Instituto colombiano para el fomento de la Educación Superior

Tajfel, H. (1984). Grupos humanos y categorías sociales: Estudios de Psicología Social. Barcelona: Herder

Turner, J. (1990). Redescubrir al Grupo social. Madrid: Morata.

Valles, M. (1999). Técnicas cualitativas de investigación social: Reflexión metodológica y práctica social. Madrid: Síntesis 